Moreover, during SARS outbreak, N95 respirators were perceived to be difficult to tolerate by healthcare workers due to their association with impaired mental performance and headache. According to Professor Seto, the WHO has recommended the use of surgical masks for people living with infected patients and N95 respirators for aerosol-generating procedures. In 2009, the US CDC recommended the use of N95 respirators in all circumstances; however, with evolution of coronavirus pandemics, the guidelines were revised in 2010 and N95 respirators were recommended only for aerosol-generating procedures. ${ }^{6}$ With increasing global apprehension concerning the spread of coronaviruses, people are rushing to Amazon and local pharmacies to buy surgical masks and N95 respirators, resulting in a shortage. Different social media celebrities are also promoting a dangerous myth by unnecessarily using these masks. Considering that N95 respirators filter out $95 \%$ of small particles, medical professionals need to wear these masks during close contact with patients. However, they are of no use for general public because these heavy particles can be transmitted through surfaces and, to a limited extent, through air. In a recent press conferences, Alex Azar issued a statement that the United States needs $\sim 300$ million N95 respirators for healthcare workers due to the rapid increase in prevalence of this infection. ${ }^{7}$ The paucity of masks and respirators has led to significant price escalation. Some businesses in United States are completely sold out of masks despite evidence that healthy people do not need to wear them. ${ }^{8}$

According to the Centers for Disease Control and Prevention, frequent hand washing with running water and soap for at least 20 seconds, using hand sanitizer, avoiding large gatherings and crowds, and disinfecting fomites may primarily protect others from the virus. However, the eminent global shortage and high prices of masks cannot be overstated. The respective authorities and governments need to develop and implement the proper guidelines and preventative strategies and make sure that the general public is adhering to the proposed protocols. Governments should also provide accurate information and should clarify misinformation spread by social media to help the public face this pandemic.
Acknowledgments. None.

Financial support. No financial support was provided relevant to this article.

Conflicts of interest. All authors report no conflicts of interest relevant to this article.

\section{References}

1. Novel coronavirus (COVID-19) situation report-62. World Health Organization website. https://www.who.int/docs/default-source/coronaviruse/ situation-reports/20200322-sitrep-62-covid-19.pdf?sfvrsn=f7764c46_2. Published March 21, 2020. Accessed April 3, 2020.

2. Ji Y, Ma Z, Peppelenbosch MP, Pan Q. Potential association between COVID-19 mortality and healthcare resource availability. Lancet Global Health 2020;8(4):e480.

3. Geller C, Varbanov M, Duval RE. Human coronaviruses: insights into environmental resistance and its influence on the development of new antiseptic strategies. Viruses 2012;4:3044-3068.

4. bin-Reza F, Lopez Chavarrias V, Nicoll A, Chamberland ME. The use of masks and respirators to prevent transmission of influenza: a systematic review of the scientific evidence. Influenza Other Respir Viruses 2012;6: 257-267.

5. Jefferson T, Del Mar C, Dooley L, et al. Physical interventions to interrupt or reduce the spread of respiratory viruses: systematic review. BMJ 2009;339: b3675.

6. Chung JS, Ling ML, Seto WH, Ang BS, Tambyah PA. Debate on MERS-CoV respiratory precautions: surgical mask or N95 respirators? Singapore Med $\mathrm{J}$ 2014;55:294.

7. Celebrities like Gwyneth Paltrow and Kate Hudson are wearing masks to protect against the coronavirus, but they're promoting a dangerous myth. Business Insider website. https:/www.businessinsider.com/coronavirus-cele brities-wearing-masks-are-promoting-a-myth-2020-2. Published February 27, 2020. Accessed April 3, 2020.

8. Mask prices ramp up on Amazon amid coronavirus outbreak. Daily Mail website. https://www.dailymail.co.uk/news/article-8056879/Mask-prices-rampAmazon-sell-stores-amid-coronavirus-outbreak.html. Published February 28, 2020. Accessed April 3, 2020.

9. Coronavirus disease 2019 (COVID-19)—prevention and treatment. Centers for Disease Control and Prevention. https://www.cdc.gov/coronavirus/2019ncov/prepare/prevention.html. Updated April 2, 2020. Accessed April 3, 2020.

\title{
COVID-19 among medical personnel in the operating room
}

\author{
Pathum Sookaromdee ${ }^{1}$ and Viroj Wiwanitkit ${ }^{2,3,4}$ \\ ${ }^{1}$ TWS Medical Center, Bangkok Thailand, ${ }^{2}$ Dr DY Patil University, Pune, India, ${ }^{3}$ Hainan Medical University, Haikou, China and ${ }^{4}$ Chulalongkorn University, Bangkok \\ Thailand
}

To the Editor-Surgical infection is an important issue in hospital infection control; infection can occur in a patient receiving an operation. With the new coronavirus infection, COVID-19, there is a risk of nosocomial infection. Glauser ${ }^{1}$ proposed a protocol to keep COVID-19 out of hospitals. However, the infection might be carried by medical personnel. The issue of COVID-19 among

\footnotetext{
Author for correspondence: Pathum Sookaromdee, E-mail: pathumsook@gmail.com Cite this article: Sookaromdee P and Wiwanitkit V. (2020). COVID-19 among medical personnel in the operating room. Infection Control \& Hospital Epidemiology, 41: 877-878,
} https://doi.org/10.1017/ice.2020.106 medical personnel working in the operating room has not been well clarified.

Here, we provide reports from Thailand, a country with the second-most COVID-19 infections at one point in the worldwide outbreak timeline. ${ }^{2}$ As of March 27, 2020, there were 1,136 patients with COVID-19 in Thailand, and 2 of these were medical personnel working in operating rooms. These cases occurred in 2 different rural hospitals. The first case was an anesthesiologist and the second case was an internist working as a surgeon. These 2 patients had regular work rotations in operating rooms during the

(C) 2020 by The Society for Healthcare Epidemiology of America. All rights reserved. This is an Open Access article, distributed under the terms of the Creative Commons Attribution licence (http://creativecommons.org/licenses/by/4.0/), which permits unrestricted re-use, distribution, and reproduction in any medium, provided the original work is properly cited. 
presymptomatic COVID-19 period. Surveillance for possible transmission to patients and other personnel is presently under way.

In fact, COVID-19 has been sporadically reported in a patients receiving surgery, causing special attention to be focused on management techniques related to patients. ${ }^{3,4}$ However, no reports have been published on COVID-19 among members of the medical teams who practice in operating rooms. Although surgical infection studies usually focus on patients, it is important to give attention to the practitioners who work in operation room as well.

\section{Acknowledgments.}

Financial support. No financial support was provided relevant to this article.
Conflicts of interest. All authors report no conflicts of interest relevant to this article.

\section{References}

1. Glauser W. Proposed protocol to keep COVID-19 out of hospitals. CMAJ 2020;192(10):e264-e265.

2. Yasri S, Wiwanitkit V. Editorial: Wuhan coronavirus outbreak and imported case. Adv Trop Med Pub Health Int 2020;10:1-2.

3. Tian S, Hu W, Niu L, Liu H, Xu H, Xiao SY. Pulmonary pathology of earlyphase 2019 novel coronavirus (COVID-19) pneumonia in two patients with lung cancer. J Thorac Oncol 2020 Feb 28. pii: S1556-0864(20)30132-5.

4. Li X, Liu M, Zhao Q, Liu R, Zhang H, Dong M, Xu S, Zhao H, Wei S, Song Z, Chen G, Chen J. Preliminary recommendations for lung surgery during 2019 novel coronavirus disease (COVID-19) epidemic period. Zhongguo Fei $\mathrm{Ai} \mathrm{Za}$ Zhi 2020;23:133-135.

\title{
Management-supportive measures for managers of healthcare organizations during the COVID-19 epidemic
}

\author{
Reza Dehnavieh ${ }^{1}$ and Khalil Kalavani ${ }^{2}$ (1) \\ ${ }^{1}$ Faculty of Management, Kerman University of Medical Sciences Kerman, Kerman, Iran and ${ }^{2}$ Health Services Management, Faculty of Management, Kerman \\ University of Medical Sciences Kerman, Kerman, Iran
}

To the Editor-Contagious or infectious diseases are a major cause of death. ${ }^{1}$ Epidemics are a serious threat to public health and a global challenge, ${ }^{2}$ and the management of these epidemics is very difficult. In these conditions, economic, social, and health factors of the country are of utmost concern; therefore, healthcare managers must properly manage and support healthcare centers ${ }^{3}$ and use supportive measures for the organization and staff to provide the best healthcare services possible. Presenting a scientific framework for managing health centers can be very helpful. The following management-supportive practices are the most important at healthcare centers during outbreaks:

1. Engage leadership: Leadership affects the performance of physicians and nurses. Maintain effective communication with employees, pay attention to them, and listen to them effectively.

2. Choose wise motivations: Talk about the importance of staff work, appreciate their work, and provide encouragement.

3. Note work-life balance: Define a proper and balanced workload for employees. Say that optimal performance depends on enough rest and emphasize the need to re-energize.

4. Encourage peer support: Protect your staff from external pressures and from illogical or uncertain demands from patients and individuals, and promote support among colleagues.

5. Provide resources to protect employees and their mental health: Minimize risky conditions in the organization and minimize workplace stress to ensure that staff are not exposed to additional stressors.

6. Build a good community: Build the right teamwork and improve working relationships.

7. Increase employee control over their work: Clarify your expectations of employees and create an environment for team members to perform important tasks without interruption.

8. Review your achievements regularly: Talk to staff about progress and successes.

9. Cancel unnecessary meetings: Try to avoid unnecessary gatherings at work. Use video conferencing if a meeting needs to be held.

Acknowledgments.

Financial support. No financial support was provided relevant to this article.

Conflicts of interest. All authors report no conflicts of interest relevant to this article.

\section{References}

1. Dasaklis TK, Pappis CP, Rachaniotis NP. Epidemics control and logistics operations: a review. Int J Prod Econ 2012;139:393-410.

2. Fatiregun AA, Isere EE. Epidemic preparedness and management: a guide on Lassa fever outbreak preparedness plan. Nigerian Med J 2017;58:1.

3. Massaro E, Ganin A, Perra N, Linkov I, Vespignani A. Resilience management during large-scale epidemic outbreaks. Sci Rept 2018;8:1-9. 